\title{
4-D Tomographic Representation of Coronary Arteries from One Rotational X-Ray Sequence
}

\author{
Christophe Blondel ${ }^{1,2}$, Grégoire Malandain ${ }^{1}$, Régis Vaillant ${ }^{2}$, \\ Frédéric Devernay $^{3}$, Ève Coste-Manière ${ }^{1}$, and Nicholas Ayache ${ }^{1}$ \\ 1 INRIA Sophia-Antipolis, 2004 route des Lucioles, 06 \\ 902 Sophia-Antipolis, France \\ 2 General Electric Medical Systems, 283 rue de la Minière, 78530 Buc, France \\ 3 INRIA Rhône-Alpes, 655 avenue de l'Europe, 38 \\ 330 Montbonnot, France
}

\begin{abstract}
We present a complete and fully automatic method to compute a 4-D tomographic representation of coronary arteries from one single rotational monoplane X-ray sequence. The major steps of our method are the following: (1) images filtering, (2) arteries segmentation, (3) arteries matching and reconstruction, (4) parametric deformation field computation, and (5) deformation-compensated tomographic reconstruction. Steps (2) and (3) involve only a few frames, acquired at the same cardiac cycle phase, while the steps (1), (4), and (5) use the frames acquired at all cardiac cycle phases. The originality of the presented work mainly lies in the last two steps that result in a 4-D tomographic representation, allowing for the visualization of coronary arteries anatomy from any point of view, and at any cardiac cycle time. Experiments have been conducted on one synthetic data set and on 10 patient data sets.
\end{abstract}

\section{Introduction}

X-ray coronary angiography remains the "gold standard" imaging modality for diagnosing, assessing, and treating the coronary arteries diseases. In clinical routine, visualization and measurements are still performed in 2-D projection angiograms, taken from different points of view: the inability to compute $3-\mathrm{D}$ tomographic reconstructions of the coronary arteries is due to the high amplitude and high speed motion that the coronary arteries undergo because of myocardial contraction. The motivation of the presented work is to provide the physicians with quantitative $3-\mathrm{D}$ information computed from one single rotational monoplane $X$-ray sequence, acquired with a C-arm.

Aiming to gain extra information from 2-D angiograms, some approaches proposed to compute 3-D arteries centerlines from semi-interactively segmented and matched views, acquired at the same cardiac cycle time [7. There were also attempts to identify the coronary arteries 3-D motion from biplane static acquisitions [10]2]. Contrary to recent developments in CT, reconstructions cannot be performed from a reduced set of views (selected from ECG analysis) because the rotation speed of the $\mathrm{C}$-arm is much slower than $\mathrm{CT}$ gantries rotation 
speed. Some recent feasibility studies attempted to adapt the CT ECG-gated approach 9], but required an acquisition protocol that is difficult to apply to patients, because of the dose of X-ray and the amount of contrast media that were involved.

We present a complete framework to automatically compute a $3-\mathrm{D}+\mathrm{t}$ (4-D) tomographic representation of the coronary arteries from one single rotational acquisition. Overstepping the intrinsic limitations of ECG-based methods, we determine a 4-D parametric deformation model and exploit it by taking into account every available frame in a deformation-compensated 3-D tomographic reconstruction algorithm. Finally, combinating 4-D deformation field and 3-D tomographic reconstruction, we can compute $3-\mathrm{D}$ tomographic representations at any cardiac cycle time. Thus, physicians are given the ability to visualize the coronary arteries anatomy from any point of view, and at any cardiac cycle time.

In section 2, we describe the successive methods we had to develop to achieve 4-D tomographic representation. Then, results on patient data sets are presented in section 3 Finally, clinical interest and future work is discussed in section 4

\section{Methodology}

The sequence acquisition is performed with a digital flat panel cardiovascular imaging system, spinning around the patient. The trajectory of the C-arm is a rotation with Cranial/Caudal angle being constantly $0^{\circ}$ and with Left Anterior Oblique/Right Anterior Oblique angular range between $120^{\circ}$ and $180^{\circ}$. The top rotation speed of the gantry is $40 \% \mathrm{~s}$, leading to a typical acquisition time of 4 seconds and providing, in average, 100 frames.

The complete processing of the formerly described X-ray sequence is divided into five major steps: (1) all the original images are filtered, then for only a few frames, acquired at the same cardiac cycle time, (2) the 2-D arteries centerlines are segmented, (3) the segmented 2-D arteries centerlines are matched and reconstructed in 3-D, and finally, for all frames, acquired at any cardiac cycle time, (4) the 4-D deformation field is computed, and (5) the 3-D tomographic reconstruction is performed.

\subsection{Images Filtering}

Remarking that coronary arteries, observed in the images, are dark and elongated structures, we build tubes detectors filters $R_{\sigma}$, based on the image derivatives computed at various diffusion scales [6]:

$$
R_{\sigma}(M)=\min \left(\nabla I_{\sigma}\left(M+\sigma \mathbf{v}_{\sigma}\right) \cdot \mathbf{v}_{\sigma}, \nabla I_{\sigma}\left(M-\sigma \mathbf{v}_{\sigma}\right) \cdot\left(-\mathbf{v}_{\sigma}\right)\right)
$$

where $M$ is a pixel location, $\mathbf{v}_{\sigma}$ is the direction associated to the maximal second derivative (extracted from the Hessian matrix), and $\nabla I_{\sigma}$ is the gradient of the image, computed by convolution with the derivative of a 2-D Gaussian of standard deviation $\sigma$. We obtain response maps that encode, for a given point, the likelihood of belonging to arteries of given radii, belonging to the 

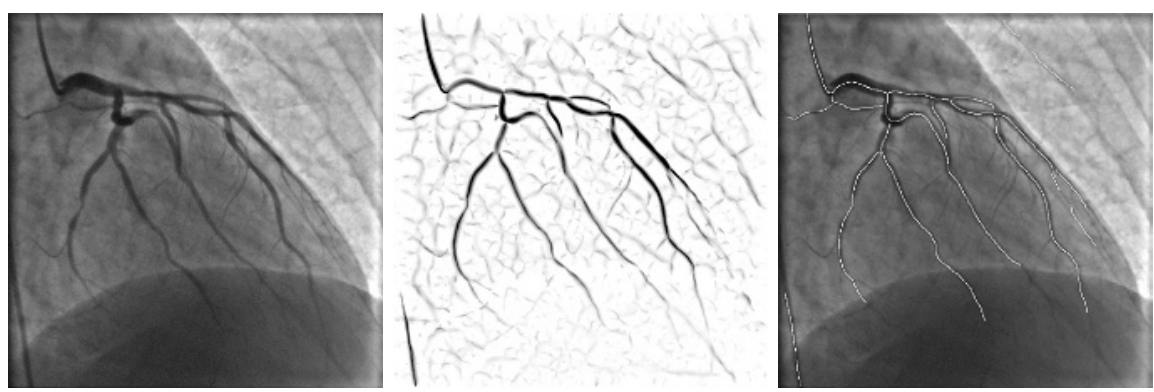

Fig. 1. From left to right: original angiogram, corresponding multiscale response map, and corresponding automatically segmented coronary arteries.

set $\Sigma=\left\{\sigma_{\min }, \ldots, \sigma_{\max }\right\}$ of targeted vessel sizes (typically $\sigma_{\min }=0.5 \mathrm{~mm}$ and $\left.\sigma_{\max }=2 \mathrm{~mm}\right)$. For every image in the X-ray sequence, we summarize the information obtained from the different detection scales in a "multiscale response" $\operatorname{map} R_{\max }: R_{\max }(M)=\max _{\sigma \in \Sigma} R_{\sigma}(M)$ (see Fig. 1).

\subsection{Coronary Arteries Centerlines 2-D Segmentation}

We select $n$ frames (at least 3, typically 5), called "reference frames", acquired at quasi the same cardiac cycle time, namely at end-diastole (when the myocardium is the most relaxed). To build a 3-D centerlines model corresponding to this "reference time" (section 2.3), we first automatically segment the coronary centerlines in the reference frames.

Centerlines are computed as local directional extrema in the multiscale response image [6]. These maxima are further binarized by hysteresis thresholding and selection of the largest connected components. They are finally linked to be collected together into a set of 2-D curves. This way, only the centerlines of long and contrasted coronary arteries are conserved (Fig 1).

\subsection{Coronary Arteries Centerlines 3-D Reconstruction}

Once the coronary arteries centerlines are segmented, we reconstruct them using a multi-ocular vision algorithm.

Using the epipolar constraint, a point belonging to the segmentation of a given reference frame $i_{1}$ can usually be matched with several points in another reference frame $i_{2}$. All the possibly matching points are reconstructed in 3-D and projected in the remaining reference frames. To disambiguate the matching, we compute a "point score" as the sum of the multiscale responses of these projected points [1]. The point score is combined with a geometrical regularizing term in a dynamic programming method to reconstruct $3-\mathrm{D}$ curves from the set of $2-\mathrm{D}$ curves in reference frame $i_{1}$. By taking all possible ordered pairs of reference frames $\left(i_{k}, i_{l}\right)$ we obtain several, partially redundant, 3 -D centerlines models. 

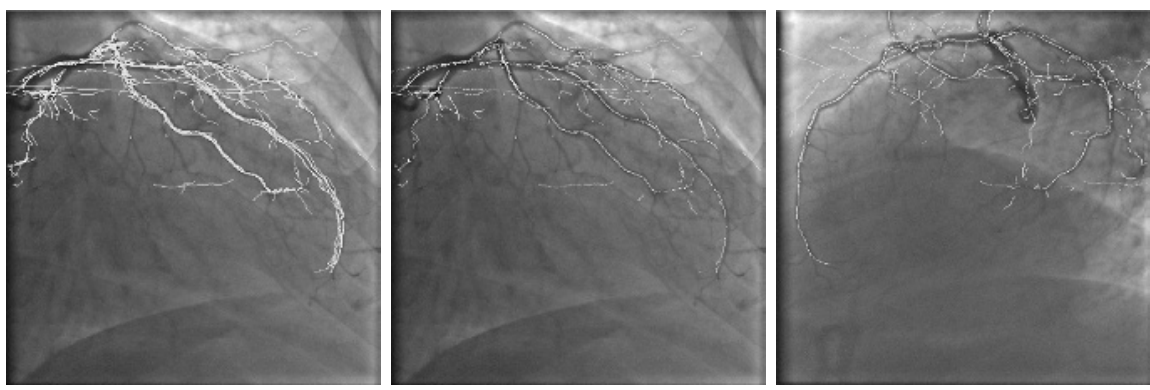

Fig. 2. From left to right: projection of redundant $3-\mathrm{D}$ reconstructed centerlines in a reference frame, projection of the fused 3-D centerlines reconstruction in the same reference frame, and in another reference frame.

However, multi-ocular reconstruction assumes that the object to be reconstructed is still in the images. This hypothesis does not strictly stand here, given that compensation has to be made for the patient's breathing movement.

As the respiratory motion mainly results in a $3-\mathrm{D}$ translation of the myocardium [12, it predominantly induces a 2-D translation of the coronary arteries in the angiograms. To compensate for the effect of respiratory motion, a bundle adjustment step [11] is performed to correct the cameras extrinsic parameters attached to the reference frames. We allow the 2-D translation of every reference frame in its camera plane. Any given 2-D translations of the reference views provide a reconstruction score, formulating how likely is the $3-\mathrm{D}$ reconstructed points superimposition with vessels in the reference frames and how smooth it is. Thus, we optimize the cameras extrinsic parameters by maximizing the overall sum of the points scores, taken as merit function, by iterating between reconstruction and bundle adjustment.

After optimization, the redundant part of the corrected 3-D centerlines models is removed by geometrically fusing the neighbor reconstructed points describing the same physical arteries (Fig. 2). To propagate the respiratory information in the entire sequence, the cameras extrinsic parameters of the intermediate frames are interpolated from the two surrounding reference frames.

\subsection{Coronary Arteries Centerlines 4-D Deformation}

The above described matching process produces a 3-D coronary arteries centerlines model, with breathing compensation, at the reference cardiac cycle time. Then, the 3-D centerlines have to be deformed using a 4-D parametric deformation field to fit the entire sequence, according to the corrected projection matrices.

4-D deformation formulation intrinsically includes temporal regularity and allows for cardiac period changes along the acquisition. On the other hand, it requires cardiac spatial repeatability, meaning that every geometrical 3-D conformation of the coronary arteries must be recovered at, potentially varying, severals time offsets, corresponding to cardiac periods. 
As the cardiac motion is cyclic, smooth, and spatially and temporally correlated, we selected as parametrization the B-solid formulation [8]. More specifically, we used a 4-D B-solid, which is a tensor product of B-splines under 3 space dimensions and 1 time dimension. This parametrization has the built-in advantage, derived from B-splines, of being a smooth, semi-local, and cyclic (when adequate knots vectors are used) representation of a 4-D deformation. To deal with 4-D deformation formulation, the cardiac cycle time is reparametrized between 0 and 1, 0 being the cardiac cycle time of the reference frames, and each frame is assigned the normalized cardiac cycle time at which it was acquired.

To determine an optimal parametric 4-D deformation, we have to exhibit the parameters vector $p^{\star}$ that maximizes the superimposition of the 3 -D centerlines model, deformed under the 4-D deformation $\Phi$, with vessels in the entire 2-D angiograms set. We build a merit function $\Psi$ that sums up, for all frames $\mathcal{I}$ and for all points in the $3-\mathrm{D}$ centerlines model $\mathcal{X}$, the multiscale response of the 2-D point obtained after deformation and projection of the original 3-D point. Additionally, a regularizing term is involved to prevent from degenerated optimal solutions. We aim to compute the optimal parametrization $p^{\star}=\operatorname{argmax} \Psi(p)$ with:

$$
\Psi(p)=\sum_{i \in \mathcal{I}} \sum_{X \in \mathcal{X}} R_{\max }^{i}\left(m_{i}\left(\Phi\left(p, X, t_{i}\right)\right)\right)+\alpha\left\|\nabla_{X} \Phi(p, . . .)\right\|
$$

where $p$ is the deformation parametrization vector, $t_{i}$ is the normalized cardiac cycle time associated to frame $i, \Phi\left(p, X, t_{i}\right)$ is the position at time $t_{i}$ of the 3 -D point being at position $X$ at time $0, m_{i}$ is the corrected projection matrix associated to frame $i, R_{\max }^{i}$ is the multiscale response of frame $i$, and $\alpha$ is the weight of a regularizing term that aims to minimize the spatial gradient of the B-solid.

Typically, we allow between 6 and 15 degrees of freedom for each space and time dimension (one degree of freedom being a 3-D deformation vector located at one 4-D control point). The optimization problem is, therefore, of very large scale (tenths of thousands of variables), and, furthermore, the merit function is non-linear. To solve numerically this optimization problem, we use the PolakRibière variant of the conjugate gradient optimization algorithm [4].

\subsection{Coronary Arteries 3-D Tomographic Reconstruction}

We take advantage of the coronary arteries 4-D deformation precomputation and incorporate it into a deformation-compensated tomographic algorithm.

Priorly, we have to process the X-ray sequence so that it complies with tomographic conditions: since the physiological acquisition conditions cannot be recovered, a mask acquisition (without contrast agent injection) is infeasible, thus available data are not subtracted from background objects, such as ribs, vertebraes, or diaphragm. We compute artificial mask images using the multiscale response maps and morphological operators (see Fig. 3). Then, we derive a logarithmic subtracted data set, that we actually use to compute the 3-D tomographic reconstruction. To comply with the classical tomographic algorithms assumptions, we suppose that the attenuation coefficients of the physical 3-D points 

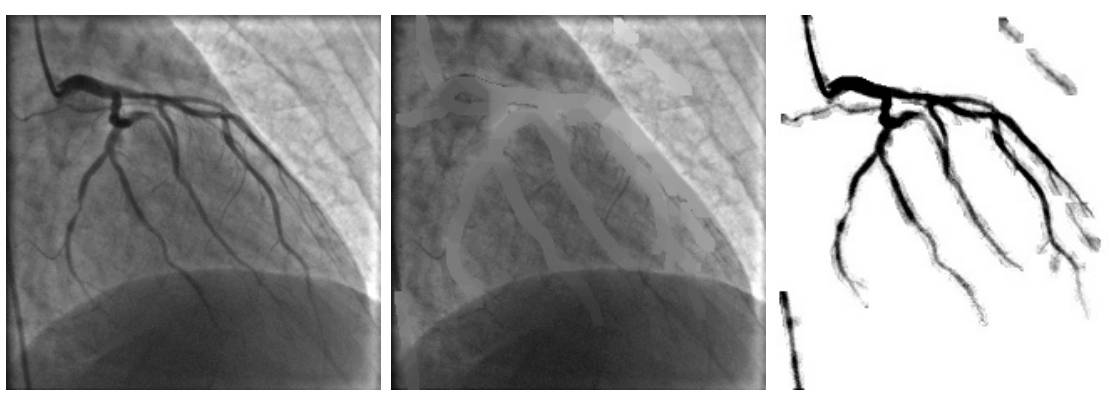

Fig. 3. From left to right: original angiogram, corresponding artificially computed mask image, and logarithmic substracted image.

are constant along the acquisition, which practically means that contrast agent filling has to be maintained as uniform as possible over acquisition time.

In a tomographic context, where we have to compute the linear attenuation coefficients $f$ in a voxels model from the subtracted sinogram measures $d$, the voxels-to-pixels contribution matrix $R$, corresponding to the projection operator, now depends on the previously computed optimal 4 -D deformation $\Phi\left(p^{\star}, .,.\right)$. Thus, we have to solve:

$$
R_{\Phi\left(p^{\star}, . . .\right)} \cdot f=d
$$

We implemented a modified additive Algebraic Reconstruction Technique (ART) algorithm [5] with binary contribution matrices: a voxel contributes to one pixel only. More precisely, a voxel $X$ (with center $C_{X}$ ), in the 3 -D volume to be reconstructed, contributes to the pixel closest to $m_{i}\left(\Phi\left(p^{\star}, C_{X}, t_{i}\right)\right)$. We chose the ART solving method for its robustness with respect to noise, computational efficiency, and adequacy to our acquisition conditions.

Finally, once a 3-D reconstruction has been computed, it can be deformed at any cardiac cycle time, using the pre-computed 4-D deformation field. Thus, the coronary arteries anatomy can be visualized from any point of view, at any cardiac cycle time.

\section{Results and Applications}

We implemented the complete method in $\mathrm{C}++$ language under Linux, and parallelized the 4-D deformation field computation step and the deformationcompensated 3-D tomographic reconstruction step, using PVM [3].

To first validate our approach, we extensively tested our approach on a dynamic synthetic data set, consisting of arteries-like tubes, moving under a beating-like motion. Deformation-compensated reconstructions had no motion artifacts, which showed that our formulation is well adapted to address the cardiac motion problem, in the X-ray coronary angiography context.

Then, we applied our method to 10 patient data sets. The tests confirmed that motion compensation and artificial subtraction produce relevant 3-D tomographic reconstructions of beating coronary arteries. The quality of the $3-\mathrm{D}$ to- 

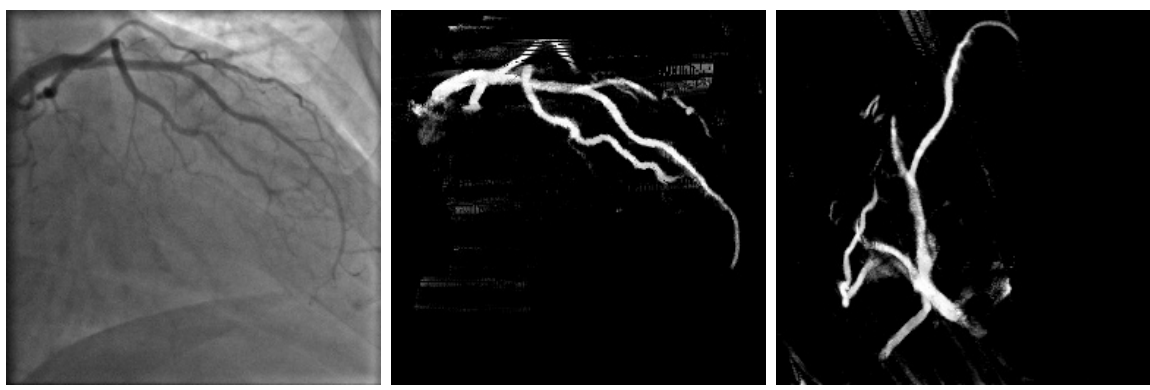

Fig. 4. From left to right: original angiogram, Maximum Intensity Projection of the reconstruction viewed under the same orientation, but at a different cardiac cycle time, and Maximum Intensity Projection of the reconstruction viewed at the same cardiac cycle time, but under a different (mechanically non-reachable) orientation.

mographic reconstructions allowed for the sectional profiling of the major vessels and for the visualization of up to third order vessels.

Using simultaneously the 3-D tomographic reconstruction and the 4-D deformation field, we have a 4-D representation of the coronary arteries allowing for visualization from any point of view, and at any cardiac cycle time (Fig. 4). In particular, the physician can visualize the coronary arteries from mechanically non-reachable points of view. He/she also can observe the 4-D beating coronary arteries from any static or moving point of view.

Typical computational times are the following: on a Pentium III at $1 \mathrm{GHz}$ workstation, images filtering takes 3 minutes, coronary arteries segmentation, matching and bundle adjustment takes 20 minutes in average, and on 8 clustered Pentium III at $933 \mathrm{MHz}$ workstations, 4-D deformation field computation takes 1 hour, and deformation-compensated 3-D reconstruction takes 1 hour.

\section{Discussion and Perspectives}

We proposed a complete method to automatically compute 3-D tomographic reconstructions at any cardiac cycle time from one single rotational X-ray angiography sequence (acquired with a C-arm, which is a widely available imaging device). New clinical applications become available from 4-D deformation field computation and 3-D tomographic reconstruction. For instance, they should allow for better understanding of cardiac motion (especially the assessment of the correlation between pathologies and arteries flexion during the cardiac cycle [2]), better visualization of coronary arteries anatomy, and offer the capacity of performing truly 3-D quantitative measurements of anatomical parameters, such as stenosis severity, that are crucial for diagnosis and treatment of patients.

To be successfully applicable on a daily base in a clinical context, our method demands some critical acquisition features to be fulfilled: constant contrast agent filling, cardiac spatial repeatability, and large angular range. In addition, the computational cost of our method needs to be reduced.

Our future trends are to process more patient data sets to make the method adequate for routine clinical use and to speed up the algorithms implementation. 


\section{References}

1. C. Blondel, R. Vaillant, F. Devernay, G. Malandain, and N. Ayache. Automatic trinocular 3D reconstruction of coronary artery centerlines from rotational x-ray angiography. In Proc. of CARS, pages 832-837, Paris, 2002. Springer.

2. Z. Ding and M.H. Friedman. Quantification of 3-D coronary arterial motion using clinical biplane cineangiograms. International Journal of Cardiac Imaging, 16(5): 331-346, 2000.

3. A. Geist, A. Beguelin, J.J. Dongarra, W. Jiang, R. Manchek, and V.S. Sunderam. PVM 3 user's guide and reference manual. Technical Report ORNL/TM-12187, Oak Ridge National Laboratory, 1993.

4. P.E. Gill, W. Murray, and M.H. Wright. Practical optimization. Academic Press, 1982.

5. G. Herman. Image reconstruction from projections. Academic Press, 1980.

6. K. Krissian, G. Malandain, N. Ayache, R. Vaillant, and Y. Trousset. Model-based detection of tubular structures in 3D images. Computer Vision and Image Understanding, 80(2): 130-171, 2000.

7. F. Mourgues, F. Devernay, G. Malandain, and È. Coste-Manière. 3D+t modeling of coronary artery tree from standard non simultaneous angiograms. In Medical Image Computing and Computer-Assisted Intervention, volume 2208 of LNCS, pages 1320-1322. Springer, 2001.

8. P. Radeva, A. Amini, and J. Huang. Deformable B-solids and implicit snakes for 3D localization and tracking of SPAMM MRI data. Computer Vision and Image Understanding, 66(2): 163-178, 1997.

9. V. Rasche, M. Grass, R. Koppe, A. Bücker, R.W. Günther, H. Kühl, J. Op de Beek, R. Bertram, and R. Suurmond. ECG-gated 3D rotational coronary angiography. In Proc. of $C A R S$, pages 826-831, Paris, 2002. Springer.

10. G. Shechter, F. Devernay, È. Coste-Manière, and E. R. McVeigh. Temporal tracking of 3D coronary arteries in projection angiograms. In Proc. of SPIE Medical Imaging, 2002.

11. B. Triggs, P. McLauchlan, R. Hartley, and A. Fitzgibbon. Vision Algorithms: Theory and Practice, volume 1883 of LNCS, chapter Bundle Adjustment - A Modern Synthesis, pages 298-375. Springer, 2000.

12. Y. Wang, S.J. Riederer, and R.L. Ehman. Respiratory motion of the heart: Kinematics and the implications for the spatial resolution in coronary imaging. Magnetic Resonance in Medicine, 33(5): 713-719, 1995. 